Intensivmed 2009 · 46:524

DOI 10.1007/s00390-009-0123-3

Online publiziert: 5. November 2009

(C) Springer-Verlag 2009

\author{
L. Engelmann \\ Einheit für Multidisziplinäre Intensivmedizin (EMI), \\ Universitätsklinikum Leipzig AöR
}

\title{
Neue Rubrik: Berufspolitisches Forum
}

Die Neugestaltung der „Intensivmedizin und Notfallmedizin“ hat uns zu einem „Berufspolitischen Forum“ verholfen - eine Rubrik, die aus der Sicht des Rubrikenverantwortlichen

1. die Intensivmedizin in gesamtgesellschaftliche Zusammenhänge einordnen soll. Es gibt Verlautbarungen der Politik, aber auch verwandter Fachgesellschaften wie unserer Muttergesellschaft, der DGIM, die unsere Arbeit tangieren und nachhaltig beeinflussen. Die meist längeren Texte sollen hier in komprimierter Form dargestellt und bewertet werden.

2. vor allem aber auch die Sorgen und Nöte der Intensivmediziner in Richtung Politik und Gesellschaft kanalisiert. Die Rolle der Intensivmedizin wird immer bedeutsamer, ihr Anteil an der Gesamtbettenzahl wächst. Ihr Budget ist, gemessen an den verfügbaren Betten, überproportional. Man sollte seriöse Kosten-Nutzen-Analysen erwarten. Aber weit gefehlt, meist inkompetente Verwaltungsangehörige schreiben vor, was der Intensivmediziner zu tun und zu lassen hat. Die Vorgaben gehen nur in Richtung Kosten-, in der Regel Personalreduktion, während der Intensivmediziner in seiner Verantwortung für Behandlungsqualität allein gelassen wird und in die Ecke des Übernahmeverschuldens gedrängt wird.
3. eine Plattform für Antworten darstellen soll, die die Position der DGIIN zu den in der Praxis drängenden Fragen enthält, unabhängig davon, ob die Antworten gesetzlich fundiert sind, Richtlinien- oder Empfehlungscharakter haben oder nur die Position der DGIIN darstellen. In den letzten Monaten hat es eine Reihe von Anfragen an die Gesellschaft gegeben, die sich mit personeller Ausstattung, Qualitätssicherung, Arbeitsorganisation, Zertifizierung und Therapiebegrenzung befassten. Die DGIIN ist die Antworten zumeist schuldig geblieben. Das wird sich nun ändern.

Es liegt nahe, dass der Rubrikenverantwortliche zugleich Sekretär der DGIIN ist, um bei den teils kontroversen Antworten, die Meinung des Vorstandes abzubilden. Die internistische Intensivmedizin muss heraus aus ihrer Dulderrolle und sich zu den brennenden Fragen der täglichen Praxis permanent artikulieren. Das betrifft auch die Zusammenarbeit mit den Schwerpunktgesellschaften, die Mitwirkung der internistischen Intensivmedizin in der Notaufnahme, vor allem aber die Anerkennung des sich immer noch rasch entwickelnden Fachgebietes und sich daraus ergebender Konsequenzen durch die Krankenhausadministration und die Politik. Es sollten Möglichkeiten diskutiert werden, die Intensivmedizin in der tatsächlichen Kosten-Erlös-Relation zu betrachten und dabei die Arbeitsqualität zu fördern.
Die Mitglieder der Gesellschaft und die Leser der Zeitschrift sind aufgefordert, die Rubrik mit Leben zu füllen und ihr Aktualität und Spannung zu geben.

Prof. Dr. L. Engelmann

Generalsekretär der DGIIN

\section{Korrespondenzadresse}

Prof. Dr. L. Engelmann

Einheit für Multidisziplinäre Intensivmedizin (EMI), Universitätsklinikum Leipzig AöR Liebigstr. 20, 04103 Leipzig

lothar.engelmann@medizin.uni-leipzig.de 